hep-ph/0412029

\title{
Three-flavour effects and CP- and T-violation in neutrino oscillations
}

\author{
E. Kh. Akhmedov* \\ AHEP Group, Instituto de Física Corpuscular - C.S.I.C. \\ Universitat de València, Edificio Institutos de Paterna \\ Apt 22085, E-46071 València, Spain
}

\begin{abstract}
Some theoretical aspects of 3-flavour (3f) neutrino oscillations are reviewed. These include: general properties of 3 f oscillation probabilities; matter effects in $\nu_{\mu} \leftrightarrow \nu_{\tau}$ oscillations; 3 effects in oscillations of solar, atmospheric, reactor and supernova neutrinos and in accelerator long-baseline experiments; $\mathrm{CP}$ and $\mathrm{T}$ violation in neutrino oscillations in vacuum and in matter, and the problem of $U_{e 3}$.
\end{abstract}

Pacs numbers: 14.60.Pq, 14.60.Lm, 26.65+t

* On leave from National Research Centre Kurchatov Institute, Moscow 123182, Russia. Present address: ICTP, Strada Costiera 11, 34014 Trieste, Italy. E-mail: akhmedov@ictp.trieste.it 


\section{Introduction}

Explanation of the solar, atmospheric, reactor and accelerator neutrino data [1] (with the exception of the LSND result, which still awaits its confirmation) in terms of neutrino oscillations requires at least three neutrino species, and in fact three neutrino species are known to exist $-\nu_{e}, \nu_{\mu}$ and $\nu_{\tau}$. Yet, until a few years ago most studies of neutrino oscillations were performed in the 2-flavour framework. There were essentially two reasons for that: (i) simplicity - there are much fewer parameters in the 2-flavour case than in the 3-flavour one, and the expressions for the transition probabilities are much simpler and by far more tractable, and (ii) the hierarchy $\Delta m_{\text {sol }} \ll \Delta m_{\text {atm }}$ and the smallness of the leptonic mixing parameter $\left|U_{e 3}\right|$, which allow to effectively decouple different oscillation channels. The 2flavour approach indeed proved to be a good first approximation, which is a consequence of the above point (ii).

However, the increased accuracy of the available and especially of expected neutrino data makes it very important to take even relatively small effects in neutrino oscillations into account. As we shall see, 3-flavour (3f) effects can lead to corrections up to $\sim 10 \%$ to 2-flavour oscillation probabilities, which is comparable to the accuracy of the present-day neutrino data. In addition, effects specific to $\geq 3$ flavour neutrino oscillations, such as CP and $\mathrm{T}$ violation, are of great interest and being widely discussed now. All this makes $3 \mathrm{f}$ analyses of neutrino oscillations mandatory.

In my talk some theoretical issues pertaining to $3 f$ neutrino oscillations are reviewed. The topics that are discussed include: general properties of $3 f$ oscillation probabilities; matter effects in $\nu_{\mu} \leftrightarrow \nu_{\tau}$ oscillations; 3f effects in oscillations of solar, atmospheric, reactor and supernova neutrinos and in accelerator long-baseline experiments; $\mathrm{CP}$ and $\mathrm{T}$ violation in neutrino oscillations in vacuum and in matter; the problem of $U_{e 3}$.

\section{Leptonic mixing and neutrino oscillations}

The leptonic mixing matrix $U$ connects neutrino flavour eigenstates $\left|\nu_{a}\right\rangle(a=e, \mu, \tau)$ with the mass eigenstates $\left|\nu_{i}\right\rangle(i=1,2,3)$ :

$$
\left|\nu_{a}\right\rangle=\sum_{i} U_{a i}^{*}\left|\nu_{i}\right\rangle
$$

In the $3 \mathrm{f}$ case the leptonic mixing matrix $U$ is a unitary $3 \times 3$ matrix, which in the standard parameterization can be written as

$$
\begin{aligned}
U & =O_{23} \Gamma_{\delta} O_{13} \Gamma_{\delta}^{\dagger} O_{12} \\
& =\left(\begin{array}{ccc}
c_{12} c_{13} & s_{12} c_{13} & s_{13} \mathrm{e}^{-\mathrm{i} \delta_{\mathrm{CP}}} \\
-s_{12} c_{23}-c_{12} s_{13} s_{23} \mathrm{e}^{\mathrm{i} \delta_{\mathrm{CP}}} & c_{12} c_{23}-s_{12} s_{13} s_{23} \mathrm{e}^{\mathrm{i} \delta_{\mathrm{CP}}} & c_{13} s_{23} \\
s_{12} s_{23}-c_{12} s_{13} c_{23} \mathrm{e}^{\mathrm{i} \delta_{\mathrm{CP}}} & -c_{12} s_{23}-s_{12} s_{13} c_{23} \mathrm{e}^{\mathrm{i} \delta_{\mathrm{CP}}} & c_{13} c_{23}
\end{array}\right) .
\end{aligned}
$$


Here $O_{i j}$ is the orthogonal rotation matrix in the $i j$-plane which depends on the mixing angle $\theta_{i j}, \Gamma_{\delta}=\operatorname{diag}\left(1,1, \mathrm{e}^{\mathrm{i} \delta_{\mathrm{CP}}}\right), \delta_{\mathrm{CP}}$ being the Dirac-type CP-violating phase, $s_{i j} \equiv \sin \theta_{i j}$ and $c_{i j} \equiv \cos \theta_{i j}$. In the $3 \mathrm{f}$ case there are also, in general, two Majorana-type CP-violating phases; however, these phases do not affect neutrino oscillations, and I will not discuss them.

Neutrino data allow two different neutrino mass orderings, the normal mass hierarchy and the inverted mass hierarchy (see Figs. 1 and 2). The lines of the matrix $U$ in Eq. (2)

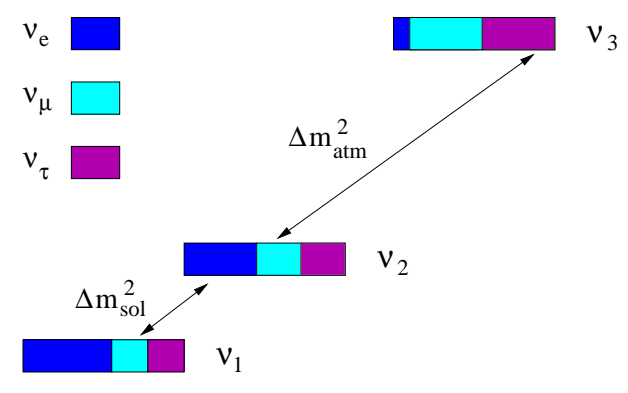

Figure 1: Normal mass hierarchy.

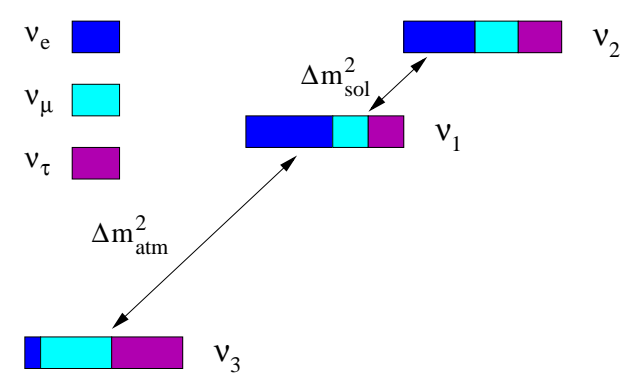

Figure 2: Inverted mass hierarchy.

represent the neutrino flavour eigenstates in terms of mass eigenstates, whereas its columns give the mass eigenstates in terms of flavour eigenstates. In particular, the value of $\left|U_{e 3}\right|^{2}$ is the weight of $\nu_{e}$ in the third mass eigenstate.

\section{Three-flavour neutrino oscillations in matter}

Neutrino oscillations in matter are described by the Schrödinger-like evolution equation

$$
i \frac{d}{d t}\left(\begin{array}{c}
\nu_{e} \\
\nu_{\mu} \\
\nu_{\tau}
\end{array}\right)=\left[U\left(\begin{array}{ccc}
E_{1} & 0 & 0 \\
0 & E_{2} & 0 \\
0 & 0 & E_{3}
\end{array}\right) U^{\dagger}+\left(\begin{array}{ccc}
V(t) & 0 & 0 \\
0 & 0 & 0 \\
0 & 0 & 0
\end{array}\right)\right]\left(\begin{array}{c}
\nu_{e} \\
\nu_{\mu} \\
\nu_{\tau}
\end{array}\right) .
$$

Here $E_{i}$ are the neutrino eigenenergies in vacuum, and the effective potential $V=\sqrt{2} G_{F} N_{e}$ is due to the charged-current interaction of $\nu_{e}$ with the electrons of the medium [2]. The 
neutral current induced potentials are omitted from Eq. (3) because they are the same for neutrinos of all three species and therefore do not affect neutrino oscillations. This, however, is only true in leading (tree) order; radiative corrections induce tiny differences between the neutral current potentials of $\nu_{e}, \nu_{\mu}$ and $\nu_{\tau}$ and, in particular, result in a very small $\nu_{\mu}-\nu_{\tau}$ potential difference $V_{\mu \tau} \sim 10^{-5} V$ [3]. This quantity is negligible in most situations but may be important for supernova neutrinos.

For matter of constant density, closed-form solutions of the evolution equation can be found 4]; however, the corresponding expressions are rather complicated and not easily tractable. For a general electron density profile $N_{e} \neq$ const no closed-form solutions exist. It is therefore desirable to have approximate analytic solutions of the neutrino evolution equation. A number of such solutions were found, most of them based on the expansions in one (or both) of the two small parameters: $\Delta m_{21}^{2} / \Delta m_{31}^{2}=\Delta m_{\mathrm{sol}}^{2} / \Delta m_{\mathrm{atm}}^{2} \simeq 0.03,\left|U_{e 3}\right|=$ $\left|\sin \theta_{13}\right| \lesssim 0.2$ [1]. For a recent discussion and a summary of the earlier results see ref. [5].

In the limits $\Delta m_{21}^{2}=0$ or $U_{e 3}=0$ the transition probabilities acquire an effective 2-flavour (2f) form. When both these parameters vanish, the genuine $2 \mathrm{f}$ case is recovered.

\section{General properties of $3 f$ oscillation probabilities}

In the $3 f$ case, there are nine oscillation (survival and transition) probabilities for neutrinos and the same number for antineutrinos, hence altogether 18 probabilities. How many of them are independent?

Consider first only neutrinos. Unitarity (probability conservation) gives 6 constraints

$$
\sum_{b} P_{a b}=\sum_{a} P_{a b}=1 \quad(a, b=e, \mu, \tau),
$$

of which 5 are independent; this leaves $9-5=4$ oscillation probabilities independent. However, recently it has been realized [5] that one can further reduce the number of independent oscillation probabilities. This is possible because the evolution equation (3) has a symmetry related to the fact that the matrix of matter-induced potentials $\operatorname{diag}(V(t), 0,0)$ commutes with $O_{23}$. Let us define the " $\theta_{23}$-transformed" probabilities

$$
\tilde{P}_{a b}=P_{a b}\left(s_{23}^{2} \leftrightarrow c_{23}^{2}, \sin 2 \theta_{23} \rightarrow-\sin 2 \theta_{23}\right)
$$

(this transformation can, e.g., be achieved through the shift $\theta_{23} \rightarrow \theta_{23}+\pi / 2$ ). Then, by inspecting the properties of Eq. (3) with respect to the rotation by $\mathrm{O}_{23}$, it is easy to show that

$$
P_{e \tau}=\tilde{P}_{e \mu}, \quad P_{\tau \mu}=\tilde{P}_{\mu \tau}, \quad P_{\tau \tau}=\tilde{P}_{\mu \mu} .
$$

Two of these three relations are independent, which leaves us with $4-2=2$ independent probabilities. Not any two probabilities would do; one possible choice is $P_{e \mu}$ and $P_{\mu \tau}$. 
Since the oscillation probabilities for antineutrinos $P_{\bar{a} \bar{b}}$ are related to those for neutrinos $P_{a b}$ through

$$
P_{\bar{a} \bar{b}}=P_{a b}\left(\delta_{\mathrm{CP}} \rightarrow-\delta_{\mathrm{CP}}, V \rightarrow-V\right),
$$

one concludes that all 18 neutrino and antineutrino probabilities can be expressed through just two [5].

Analogously, by rotating Eq. (3) with the matrix $O_{23}^{\prime}=O_{23} \times \operatorname{diag}\left(1,1, e^{i \delta_{\mathrm{CP}}}\right)$, one can study the general dependence of the oscillation probabilities on the CP-violating phase $\delta_{\mathrm{CP}}$ 6]. For example, for $P_{e \mu}$ and $P_{\mu \tau}$ one finds

$$
\begin{aligned}
& P_{e \mu}=A_{e \mu} \cos \delta_{\mathrm{CP}}+B_{e \mu} \sin \delta_{\mathrm{CP}}+C_{e \mu}, \\
& P_{\mu \tau}=A_{\mu \tau} \cos \delta_{\mathrm{CP}}+B_{\mu \tau} \sin \delta_{\mathrm{CP}}+C_{\mu \tau}+D_{\mu \tau} \cos 2 \delta_{\mathrm{CP}}+E_{\mu \tau} \sin 2 \delta_{\mathrm{CP}} .
\end{aligned}
$$

\section{3 effects in neutrino oscillations}

\subsection{Two kinds of three-flavour effects}

There are two kinds $3 f$ effects in neutrino oscillations. First, there are effects which, in a sense, are trivial. These include:

- The existence of new physical oscillation channels - i.e., in addition to $\nu_{e} \leftrightarrow \nu_{\mu}$ there are $\nu_{e} \leftrightarrow \nu_{\tau}$ and $\nu_{\mu} \leftrightarrow \nu_{\tau}$ channels; mutual influence of the channels through unitarity;

- New "parameter channels" for the same physical channel. For example, $\nu_{e} \leftrightarrow \nu_{\mu}$ oscillations can be governed by two pairs of parameters, $\left(\Delta m_{21}^{2}, \theta_{12}\right)$ and $\left(\Delta m_{31}^{2}, \theta_{13}\right)$, corresponding to two ways in which these oscillations can occur.

Second, there are non-trivial effects, i.e. qualitatively new effects that are specific for three (or more) flavours and do not occur in the $2 \mathrm{f}$ case:

- Fundamental CP- and T-violation;

- Matter-induced T-violation;

- Interference of different "parameter channels" - specific contributions to oscillation probabilities;

- Matter effects on $\nu_{\mu} \leftrightarrow \nu_{\tau}$ oscillations.

A characteristic feature of the non-trivial $3 \mathrm{f}$ effects (except for the last one) is that they disappear if at least one mixing angle is 0 or $90^{\circ}$, or at least one $\Delta m_{i j}^{2}=0$.

I will discuss both types of $3 f$ effects. 


\subsection{Matter effects in $\nu_{\mu} \leftrightarrow \nu_{\tau}$ oscillations}

Since the matter-induced potentials for $\nu_{\mu}$ and $\nu_{\tau}$ are the same (neglecting the tiny radiative corrections), in the $2 \mathrm{f}$ case the $\nu_{\mu} \leftrightarrow \nu_{\tau}$ oscillations are not affected by matter. This, however, is not true in the $3 \mathrm{f}$ case; therefore the effect of matter on $\nu_{\mu} \leftrightarrow \nu_{\tau}$ oscillations is a pure $3 \mathrm{f}$ effect. It vanishes only when both $\Delta m_{21}^{2}$ and $U_{e 3}$ vanish. The effects of the Earth's matter on $\nu_{\mu} \leftrightarrow \nu_{\tau}$ oscillations can manifest themselves in the long-baseline accelerator as well as in atmospheric neutrino experiments [7, 8]. It has been demonstrated recently that these effects can be rather large [9].

\subsection{Solar neutrinos}

In the 3f case, solar $\nu_{e}$ can in principle oscillate into either $\nu_{\mu}$, or $\nu_{\tau}$, or some their combination. What do they actually oscillate to?

It is easy to answer this question. The smallness of the mixing parameter $\left|U_{e 3}\right|$ implies that the mass eigenstate $\nu_{3}$, separated by a large mass gap from the other two, is approximately given by

$$
\nu_{3} \simeq s_{23} \nu_{\mu}+c_{23} \nu_{\tau}
$$

and, to first approximation, does not participate in the solar neutrino oscillations. From the unitarity of the leptonic mixing matrix it then follows that the solar neutrino oscillations are the oscillations between $\nu_{e}$ and a state $\nu^{\prime}$ which is the linear combination of $\nu_{\mu}$ and $\nu_{\tau}$, orthogonal to $\nu_{3}$ :

$$
\nu^{\prime}=c_{23} \nu_{\mu}-s_{23} \nu_{\tau}
$$

Therefore for solar neutrinos

$$
P\left(\nu_{e} \rightarrow \nu_{\mu}\right) / P\left(\nu_{e} \rightarrow \nu_{\tau}\right) \simeq c_{23}^{2} / s_{23}^{2} .
$$

Since the mixing angle $\theta_{23}$, responsible for the atmospheric neutrino oscillations, is known to be close to $45^{\circ}$, Eq. (11) implies that the solar $\nu_{e}$ oscillate into a superposition of $\nu_{\mu}$ and $\nu_{\tau}$ with equal or almost equal weights. The same argument applies to the long-baseline oscillations of reactor antineutrinos (KamLAND experiment). For reactor experiments with relatively short baselines $L \simeq 1 \mathrm{~km}$ (such as $\mathrm{CHOOZ}$ and Palo Verde), the same is true when $\theta_{13} \ll 0.03$. In the opposite limit, $\theta_{13} \gg 0.03$, one finds $P\left(\bar{\nu}_{e} \rightarrow \bar{\nu}_{\mu}\right) / P\left(\bar{\nu}_{e} \rightarrow \bar{\nu}_{\tau}\right) \simeq s_{23}^{2} / c_{23}^{2}$, which is also close to unity. In the intermediate case, $\theta_{13} \sim 0.03$, deviations from unity are possible due to the interference terms in the probabilities $P\left(\bar{\nu}_{e} \rightarrow \bar{\nu}_{\mu}\right)$ and $P\left(\bar{\nu}_{e} \rightarrow \bar{\nu}_{\tau}\right)$.

What are the $3 \mathrm{f}$ effects in the oscillation probabilities of solar neutrinos? Since at low energies $\nu_{\mu}$ and $\nu_{\tau}$ are experimentally indistinguishable, all the observables depend on just one probability - the $\nu_{e}$ survival probability $P\left(\nu_{e} \rightarrow \nu_{e}\right)$. The loss of coherence of the neutrino state in the course of neutrino propagation between the Sun and the Earth leads to an effective averaging over fast oscillations due to the large mass squared difference $\Delta m_{\mathrm{atm}}^{2}=\Delta m_{31}^{2}$, which yields [10]

$$
P\left(\nu_{e} \rightarrow \nu_{e}\right) \simeq c_{13}^{4} \tilde{P}_{2 e e}\left(\Delta m_{21}^{2}, \theta_{12}, N_{\text {eff }}\right)+s_{13}^{4} .
$$


Here $\tilde{P}_{2 e e}\left(\Delta m_{21}^{2}, \theta_{12}, N_{\text {eff }}\right)$ is the 2 f survival probability of $\nu_{e}$ in matter with the effective electron density $N_{\text {eff }}=c_{13}^{2} N_{e}$.

As follows from the CHOOZ data [11, the second term in Eq. (12), $s_{13}^{4}$, does not exceed $10^{-3}$, i.e. is negligible. At the same time, the coefficient $c_{13}^{4}$ of $\tilde{P}_{2 e e}$ in the first term may differ from unity by as much as $\sim 5-10 \%$. Thus, 3 f effects may lead to an approximately energy-independent suppression of the $\nu_{e}$ survival probability by up to $10 \%$. With high precision solar data this must be taken into account.

From Eq. (12) it is clear that the fluxes of various components of the solar neutrino spectrum $f_{i}\left(i=p p,{ }^{7} \mathrm{Be},{ }^{8} \mathrm{~B}, \ldots\right)$ are always extracted from the charged-current experimental data in the combinations $f_{i} c_{13}^{4}$. This leads to an intrinsic uncertainty in their values due to the uncertainty in $\theta_{13}$. In contrast to this, the neutral currents experiments give the fluxes which are free of both astrophysics and $\theta_{13}$ uncertainties.
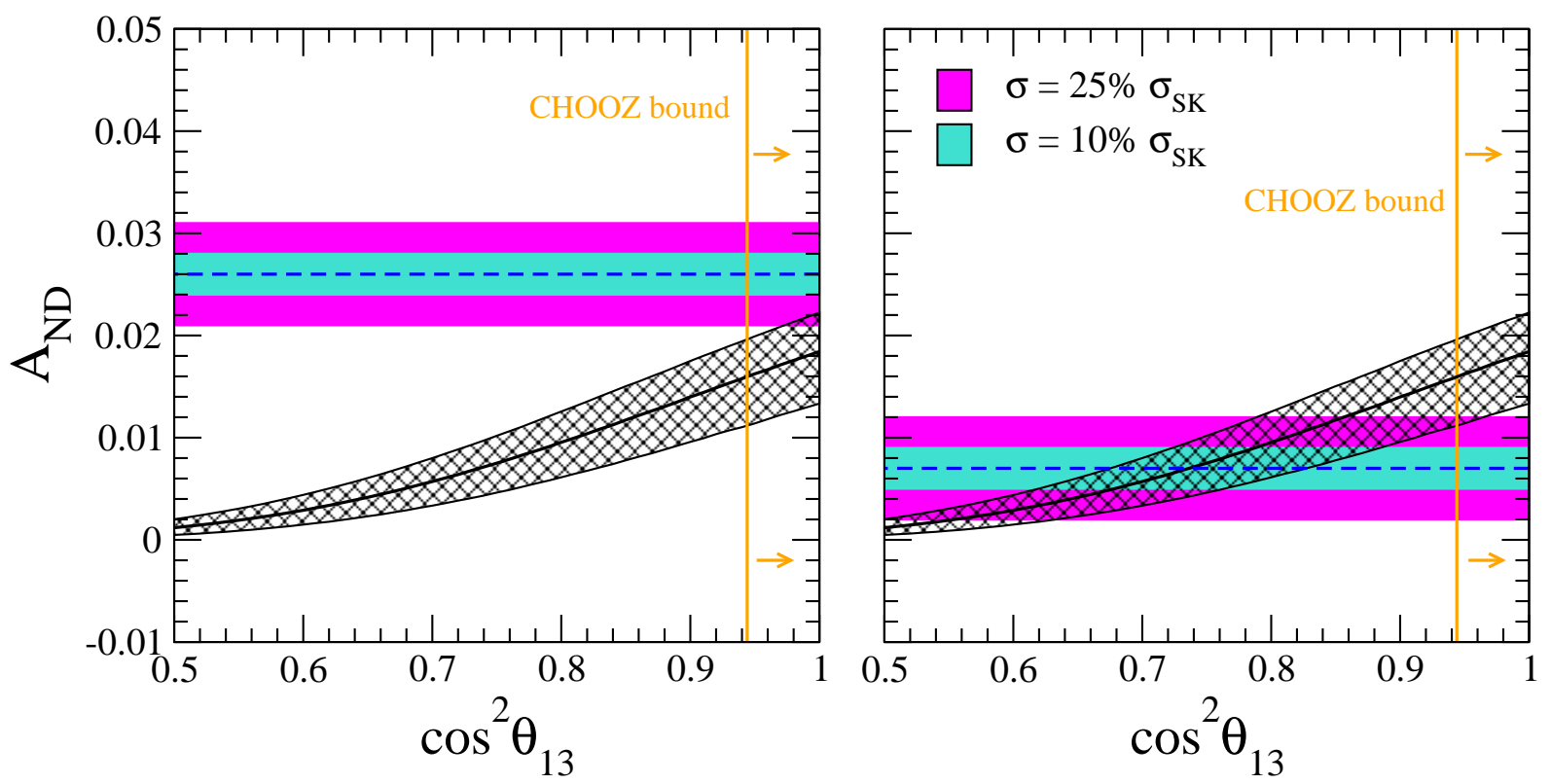

Figure 3: Expected night-day asymmetry at UNO and Hyper-Kamiokande (horizontal bands) with central values larger (left panel) and smaller (right panel) than the current SK one. Hatched areas are theoretical expectations based on $3 \sigma$ allowed regions of $\Delta m_{21}^{2}$ and $\theta_{12}$ from KamLAND and solar neutrino data. Regions allowed by CHOOZ $(3 \sigma)$ are to the right of vertical lines.

While the day-time survival probability of solar $\nu_{e}$ (12) scales essentially as $c_{13}^{4}$, the daynight signal difference due to the Earth matter effect scales as $c_{13}^{6}$ [12, 13]. Unfortunately, the experimental errors of the day-night asymmetry measured by the Super-Kamiokande (SK) and SNO detectors are too large and no useful information on $\theta_{13}$ can presently be extracted (the SK value, which has a smaller error, is $A_{N D}=2.1 \% \pm 2.0 \%$ (stat) $\pm 1.3 \%$ (syst)). However, future large water Cherenkov detectors, such as UNO or Hyper-Kamiokande, may be able to probe $\theta_{13}$ [13. This is illustrated in Fig. 3, which shows the night-day signal asymmetry $A_{N D}$ in future detectors. Fig. 3 allows for possible deviations of the central value 
of the observed asymmetry from the current SK one within $1 \sigma$ error of the latter. As one can see from the left panel, if the future central value of measured $A_{N D}$ is higher than the present SK one, the current upper limit on $\theta_{13}$ can be improved. If, on the contrary, a lower value of $A_{N D}$ is measured (right panel), the derived upper limit on $\theta_{13}$ will be substantially weaker than the current one and thus irrelevant. However, as can be seen from the figure, in that case a lower bound on $\theta_{13}$ may appear; together with the current upper bound it may actually lead to a rather precise determination of $\theta_{13}$ [13. If the future central value of $A_{N D}$ coincides with the current SK one, no useful information on $\theta_{13}$ can be obtained.

\subsection{Atmospheric neutrinos}

(1) The dominant channel $\nu_{\mu} \leftrightarrow \nu_{\tau}$. In the $2 \mathrm{f}$ limit, there are no matter effects in this channel (neglecting tiny $V_{\mu \tau}$ caused by radiative corrections). The oscillation probability is independent from the sign of $\Delta m_{31}^{2}$, i.e. cannot differentiate between the normal and inverted neutrino mass hierarchies. The $3 \mathrm{f}$ effects result in a sensitivity to matter effects and to the sign of $\Delta m_{31}^{2}$.

(2) The subdominant channels $\nu_{e} \leftrightarrow \nu_{\mu, \tau}$. Contributions of these oscillation channels to the number of $\mu$ - like events are subleading and difficult to observe. For e-like events, one could a priori expect significant oscillations effects. However, these effects are in fact strongly suppressed because of the specific composition of the atmospheric neutrino flux and proximity of the mixing angle $\theta_{23}$ to $45^{\circ}$. Indeed, in the $2 \mathrm{f}$ limits one finds

$$
\frac{F_{e}-F_{e}^{0}}{F_{e}^{0}}=\tilde{P}_{2}\left(\Delta m_{31}^{2}, \theta_{13}, V\right) \cdot\left(r s_{23}^{2}-1\right)
$$

in the limit $\Delta m_{21}^{2} \rightarrow 0[7]$, and

$$
\frac{F_{e}-F_{e}^{0}}{F_{e}^{0}}=\tilde{P}_{2}\left(\Delta m_{21}^{2}, \theta_{12}, V\right) \cdot\left(r c_{23}^{2}-1\right)
$$

in the limit $s_{13} \rightarrow 0$ [14. Here $F_{e}^{0}$ and $F_{e}$ are the $\nu_{e}$ fluxes in the absence and in the presence of the oscillations, respectively, and $r \equiv F_{\mu}^{0} / F_{e}^{0}$. At low energies $r \simeq 2$; also, we know that $s_{23}^{2} \simeq c_{23}^{2} \simeq 1 / 2$. Therefore the factors $\left(r s_{23}^{2}-1\right)$ and $\left(r c_{23}^{2}-1\right)$ in Eqs. (13) and (14) are very small and strongly suppress the oscillation effects even if the transition probabilities $\tilde{P}_{2}$ are close to unity. This happens because of the strong cancellations of the transitions from and to the $\nu_{e}$ state.

All this looks as a conspiracy to hide the oscillation effects on the e-like events! This

conspiracy is, however, broken by the $3 f$ effects. Keeping both $\Delta m_{21}^{2}$ and $s_{13}$ in leading order yields [15].

$$
\begin{aligned}
\frac{F_{e}-F_{e}^{0}}{F_{e}^{0}} \simeq \tilde{P}_{2}\left(\Delta m_{31}^{2}, \theta_{13}\right) \cdot\left(r s_{23}^{2}-1\right) & +\tilde{P}_{2}\left(\Delta m_{21}^{2}, \theta_{12}\right) \cdot\left(r c_{23}^{2}-1\right) \\
& -2 s_{13} s_{23} c_{23} r \operatorname{Re}\left(\tilde{A}_{e e}^{*} \tilde{A}_{\mu e}\right)
\end{aligned}
$$


Here $\tilde{A}_{e e}$ and $\tilde{A}_{\mu e}$ are the $\nu_{e}$ survival and transition amplitudes in the rotated basis $\tilde{\nu} \approx$

$O_{13}^{\dagger} O_{23}^{\dagger} \nu_{f l}$, where $\nu_{f l}$ is the neutrino state in the flavour basis. The interference term, which represents the genuinely $3 \mathrm{f}$ effects, is not suppressed by the flavour composition of the atmospheric neutrino flux; it can reach a few per cent and may be partially responsible for some excess of the upward-going sub-GeV e-like events observed at Super-Kamiokande. However, this term seems to be insufficient to fully explain the excess, which may be a hint of a deviation of $\theta_{23}$ from $45^{\circ}$ [15].

\subsection{Reactor antineutrinos}

For reactor neutrino experiments, the $\bar{\nu}_{e}$ survival probability can be written as

$$
P_{\bar{e} \bar{e}} \simeq 1-\sin ^{2} 2 \theta_{13} \cdot \sin ^{2}\left(\frac{\Delta m_{31}^{2}}{4 E} L\right)-c_{13}^{4} \sin ^{2} 2 \theta_{12} \cdot \sin ^{2}\left(\frac{\Delta m_{21}^{2}}{4 E} L\right)
$$

Since the average energy of reactor $\bar{\nu}_{e}$ 's is $\bar{E} \sim 4 \mathrm{MeV}$, for intermediate-baseline experiments, such as CHOOZ and Palo Verde $(L \lesssim 1 \mathrm{~km})$, one has

$$
\frac{\Delta m_{31}^{2}}{4 E} L \sim 1, \quad \frac{\Delta m_{21}^{2}}{4 E} L \ll 1 .
$$

This justifies the use of the one mass scale dominance approximation, in which the last term in (16) is neglected:

$$
P\left(\bar{\nu}_{e} \rightarrow \bar{\nu}_{e}\right)=1-\sin ^{2} 2 \theta_{13} \cdot \sin ^{2}\left(\frac{\Delta m_{31}^{2}}{4 E} L\right) .
$$

This is a pure $2 \mathrm{f}$ result. Note, however, that disregarding the last term in (16) is only legitimate if $\theta_{13}$ is larger than $\sim 0.03$, which is about the reach of the currently discussed next-generation reactor neutrino experiments.

For KamLAND, which is a very long baseline reactor experiment $(\bar{L} \simeq 170 \mathrm{~km})$, one has

$$
\frac{\Delta m_{31}^{2}}{4 E} L \gg 1, \quad \frac{\Delta m_{21}^{2}}{4 E} L \gtrsim 1 .
$$

Averaging over the fast oscillations driven by $\Delta m_{31}^{2}=\Delta m_{\text {atm }}^{2}$ yields

$$
P\left(\bar{\nu}_{e} \rightarrow \bar{\nu}_{e}\right)=c_{13}^{4} P_{2 \bar{e} \bar{e}}\left(\Delta m_{21}^{2}, \theta_{12}\right)+s_{13}^{4} .
$$

This has the same form as Eq. (12). The $2 \mathrm{f}$ survival probability $P_{2 \bar{e} \bar{e}}$ is, in first approximation, just the corresponding $\bar{\nu}_{e}$ survival probability in vacuum, which can be obtained from (18) by substituting $\theta_{13} \rightarrow \theta_{12}, \Delta m_{31}^{2} \rightarrow \Delta m_{21}^{2}$. Note, however, that matter effects in KamLAND can reach a few per cent, i.e. can be comparable with the effects of non-zero $\theta_{13}$, and so should be taken into account in $3 \mathrm{f}$ analyses. The probability (20) can differ from the 2 f probability $P_{2 \bar{e} \bar{e}}$ by up to $\sim 10 \%$. 


\subsection{Long-baseline accelerator experiments}

(1) $\nu_{\mu}$ disappearance.

3f effects can result in up to $\sim 10 \%$ corrections to the disappearance probability, mainly due to the factor $c_{13}^{4}$ in the effective amplitude of the $\nu_{\mu} \leftrightarrow \nu_{\tau}$ oscillations, $\sin ^{2}\left(2 \theta_{\mu \tau}\right)_{\text {eff }} \equiv$ $c_{13}^{4} \sin ^{2} 2 \theta_{23}$. Another manifestation of 3 -flavourness are matter effects in $\nu_{\mu} \leftrightarrow \nu_{\tau}$ oscillations. The same applies to $\nu_{\tau}$ appearance in experiments with the conventional neutrino beams. $\nu_{\mu}$ disappearance receives contributions also from the subdominant $\nu_{\mu} \leftrightarrow \nu_{e}$ oscillations.

(2) $\nu_{\mu}$ appearance at neutrino factories; $\nu_{e}$ appearance at neutrino factories and in experiments with the conventional neutrino beams.

These are driven by the $\nu_{e} \leftrightarrow \nu_{\mu, \tau}$ oscillations. There are two channels through which these subdominant oscillations can proceed - those governed by the parameters $\left(\theta_{13}, \Delta m_{31}^{2}\right)$ and $\left(\theta_{12}, \Delta m_{21}^{2}\right)$. For typical energies of the long-baseline (LBL) accelerator experiments, a few $\mathrm{GeV}$ to tens of $\mathrm{GeV}$, one finds that for $\theta_{13}$ in the range $3 \cdot 10^{-3} \lesssim \theta_{13} \lesssim 3 \cdot 10^{-2}$ the two channels compete; otherwise one of them dominates.

Unlike in the case of atmospheric neutrinos, there is no suppression of the oscillation effects on the $\nu_{e}$ flux due to the flavour composition of the original flux.

The dependence of the oscillation probabilities on the CP-violating phase $\delta_{\mathrm{CP}}$ comes from the interference terms and is a pure $3 \mathrm{f}$ effect. The $3 \mathrm{f}$ effects will be especially important for the future experiments at neutrino factories which are designed for precision measurements of neutrino parameters.

\subsection{Supernova neutrinos}

In supernovae, matter density varies in a very wide range, and the conditions for three MSW 2] resonances are satisfied (taking into account that due to radiative corrections $\left.V_{\mu \tau} \neq 0\right)$. The hierarchy $\Delta m_{21}^{2} \ll \Delta m_{31}^{2}$ leads to the approximate factorization of the transition dynamics at the resonances, so that the transitions, to first approximation, are effectively $2 \mathrm{f}$ ones. However, the observable effects of the supernova neutrino oscillations depend on the transitions between all three neutrino species [16].

Supernova neutrinos can propagate significant distances inside the Earth before reaching the detector. Matter effects on the oscillations of supernova neutrinos inside the Earth depend crucially on the sequence of the neutrino flavour conversions in the supernova which, in turn, depends on the sign of $\Delta m_{31}^{2}$ and is very sensitive to the value of the leptonic mixing parameter $U_{e 3}$. Thus, the Earth matter effects on supernova neutrinos can be used to determine the sign of $\Delta m_{31}^{2}$ and to probe $\left|U_{e 3}\right|$ down to very small values $\left(\sim 10^{-3}\right)$ [17]. The transitions due to the $\nu_{\mu}-\nu_{\tau}$ potential difference $V_{\mu \tau}$ caused by radiative corrections may have observable consequences if the originally produced $\nu_{\mu}$ and $\nu_{\tau}$ fluxes are not exactly the same 18. 
If neutrinos are Majorana particles, a combination of the MSW effect and resonance spinflavour precession (RSFP) due to the interaction of neutrino transition magnetic moments $\mu_{\nu}$ with supernova magnetic fields $B$ can result in the conversion $\nu_{e} \rightarrow \bar{\nu}_{e}$. Such a conversion would lead to the transformation of the supernova $\nu_{e}$, born in the neutronization process, into their antiparticles. This effect would have a clear experimental signature and its observation would be a smoking gun evidence for the neutrino transition magnetic moments. It would also signify the leptonic mixing parameter $\left|U_{e 3}\right|$ in excess of $10^{-2}$. The conversion mechanism is efficient if $\mu_{\nu} B_{\text {res }} \gtrsim 10^{-13} \mu_{B} \cdot 10^{9} \mathrm{G}$. In the $2 \mathrm{f}$ approach, the $\nu_{e} \rightarrow \bar{\nu}_{e}$ transition is only possible in the case of the inverted neutrino mass hierarchy [19]. However, in the full $3 \mathrm{f}$ framework one finds a new RSFP resonance to exist, due to which the $\nu_{e} \rightarrow \bar{\nu}_{e}$ conversion can occur also for the normal mass hierarchy [20]. Thus, the possibility of $\nu_{e} \rightarrow \bar{\nu}_{e}$ transitions of supernova neutrinos in the case of the normal neutrino mass hierarchy is a pure $3 \mathrm{f}$ effect.

\section{$6 \mathrm{CP}$ and $\mathrm{T}$ violation in $\nu$ oscillations in vacuum}

The probability of $\nu_{a} \rightarrow \nu_{b}$ oscillations in vacuum is given by

$$
P\left(\nu_{a}, t_{0} \rightarrow \nu_{b} ; t\right)=\left|\sum_{i} U_{b i} e^{-i E_{i}\left(t-t_{0}\right)} U_{a i}^{*}\right|^{2} .
$$

In the general case of $n$ flavours the leptonic mixing matrix $U_{a i}$ depends on $(n-1)(n-2) / 2$ Dirac-type CP-violating phases $\left\{\delta_{\mathrm{CP}}\right\}$.

Under CP transformation, neutrinos are replaced by their antiparticles $\left(\nu_{a, b} \leftrightarrow \bar{\nu}_{a, b}\right)$, which is equivalent to the complex conjugation of $U_{a i}$ :

$$
\begin{array}{ll}
\mathrm{CP}: & \nu_{a, b} \leftrightarrow \bar{\nu}_{a, b} \\
& \Leftrightarrow U_{a i} \rightarrow U_{a i}^{*} \quad\left(\left\{\delta_{\mathrm{CP}}\right\} \rightarrow-\left\{\delta_{\mathrm{CP}}\right\}\right) .
\end{array}
$$

Time reversal transformation interchanges the initial and final evolution times $t_{0}$ and $t$ in Eq. (21), i.e. corresponds to evolution "backwards in time". As follows from Eq. (21), the interchange $t_{0} \rightleftarrows t$ is equivalent to the complex conjugation of the exponential factors in the oscillation amplitude. Since the transition probability only depends on the modulus of the amplitude, this is equivalent to the complex conjugation of the factors $U_{b i}$ and $U_{a i}^{*}$, which in turn amounts to interchanging $a \rightleftarrows b$. Thus, instead of evolution "backwards in time" one can consider evolution forward in time, but between the interchanged initial and final flavours:

$$
\begin{aligned}
T: \quad & t_{0} \rightleftarrows t \Leftrightarrow \nu_{a} \leftrightarrow \nu_{b} \\
& \Rightarrow U_{a i} \rightarrow U_{a i}^{*}\left(\left\{\delta_{\mathrm{CP}}\right\} \rightarrow-\left\{\delta_{\mathrm{CP}}\right\}\right) .
\end{aligned}
$$

Under the combined action of $\mathrm{CP}$ and $\mathrm{T}$ one has

$$
\begin{aligned}
\mathrm{CPT}: & \nu_{a, b} \leftrightarrow \bar{\nu}_{a, b} \text { and } t_{0} \rightleftarrows t\left(\nu_{a} \leftrightarrow \nu_{b}\right) \\
& \Rightarrow P\left(\nu_{a} \rightarrow \nu_{b}\right) \rightarrow P\left(\bar{\nu}_{b} \rightarrow \bar{\nu}_{a}\right) .
\end{aligned}
$$


From CPT invariance it follows that $\mathrm{CP}$ violation implies $\mathrm{T}$ violation and vice versa.

$\mathrm{CP}$ and $\mathrm{T}$ violation can be characterized by the probability differences

$$
\begin{gathered}
\Delta P_{a b}^{\mathrm{CP}} \equiv P\left(\nu_{a} \rightarrow \nu_{b}\right)-P\left(\bar{\nu}_{a} \rightarrow \bar{\nu}_{b}\right), \\
\Delta P_{a b}^{\mathrm{T}} \equiv P\left(\nu_{a} \rightarrow \nu_{b}\right)-P\left(\nu_{b} \rightarrow \nu_{a}\right) .
\end{gathered}
$$

From CPT invariance it follows that the CP- and T-violating probability differences coincide, and that the survival probabilities have no CP asymmetry:

$$
\Delta P_{a b}^{\mathrm{CP}}=\Delta P_{a b}^{\mathrm{T}} ; \quad \Delta P_{a a}^{\mathrm{CP}}=0 .
$$

$\mathrm{CP}$ and $\mathrm{T}$ violations are absent in the $2 \mathrm{f}$ case, so any observable violation of these symmetries in neutrino oscillations in vacuum would be a pure $\geq 3 \mathrm{f}$ effect.

In the $3 \mathrm{f}$ case, there is only one CP-violating Dirac-type phase $\delta_{\mathrm{CP}}$ and so only one CP-odd (and T-odd) probability difference:

$$
\Delta P_{e \mu}^{\mathrm{CP}}=\Delta P_{\mu \tau}^{\mathrm{CP}}=\Delta P_{\tau e}^{\mathrm{CP}} \equiv \Delta P
$$

where

$$
\Delta P=-4 s_{12} c_{12} s_{13} c_{13}^{2} s_{23} c_{23} \sin \delta_{\mathrm{CP}}\left[\sin \left(\frac{\Delta m_{12}^{2}}{2 E} L\right)+\sin \left(\frac{\Delta m_{23}^{2}}{2 E} L\right)+\sin \left(\frac{\Delta m_{31}^{2}}{2 E} L\right)\right] .
$$

It vanishes

- when at least one $\Delta m_{i j}^{2}=0$

- when at least one $\theta_{i j}=0$ or $90^{\circ}$

- when $\delta_{\mathrm{CP}}=0$ or $180^{\circ}$

- in the averaging regime

- in the limit $L \rightarrow 0\left(\right.$ as $\left.L^{3}\right)$

Clearly, this quantity is very difficult to observe.

\section{$7 \quad$ CP- and T-odd effects in $\nu$ oscillations in matter}

For neutrino oscillations in matter, CP transformation (substitution $\nu_{a} \leftrightarrow \bar{\nu}_{a}$ ) implies not only complex conjugating the leptonic mixing matrix, but also flipping the sign of the matter-induced neutrino potentials:

$$
\begin{aligned}
\mathrm{CP}: \quad U_{a i} \rightarrow & U_{a i}^{*}\left(\left\{\delta_{\mathrm{CP}}\right\} \rightarrow-\left\{\delta_{\mathrm{CP}}\right\}\right), \\
& V(r) \rightarrow-V(r) .
\end{aligned}
$$


It can be shown [21] that in matter with an arbitrary density profile, just as well as in vacuum, the action of time reversal on neutrino oscillations is equivalent to interchanging the initial and final neutrino flavours. It is also equivalent to complex conjugating $U_{a i}$ and replacing the matter density profile by the reverse one:

$$
\begin{gathered}
\mathrm{T}: \quad U_{a i} \rightarrow U_{a i}^{*}\left(\left\{\delta_{\mathrm{CP}}\right\} \rightarrow-\left\{\delta_{\mathrm{CP}}\right\}\right), \\
V(r) \rightarrow \tilde{V}(r) .
\end{gathered}
$$

Here

$$
\tilde{V}(r)=\sqrt{2} G_{F} \tilde{N}(r),
$$

$\tilde{N}(r)$ being the reverse profile, i.e. the profile that corresponds to the interchanged positions of the neutrino source and detector. In the case of symmetric matter density profiles (e.g., matter of constant density), $\tilde{N}(r)=N(r)$.

An important point is that the very presence of matter (with unequal numbers of particles and antiparticles) violates $\mathrm{C}, \mathrm{CP}$ and $\mathrm{CPT}$, leading to $\mathrm{CP}$-odd effects in neutrino oscillations even in the absence of the fundamental CP-violating phases $\left\{\delta_{\mathrm{CP}}\right\}$. This fake (extrinsic) CP violation may complicate the study of the fundamental (intrinsic) one.

\subsection{CP-odd effects in matter}

Unlike in vacuum, CP-odd effects in neutrino oscillations in matter exist even in the $2 \mathrm{f}$ case (in the case of three or more flavours, even when all $\left\{\delta_{\mathrm{CP}}\right\}=0$ ):

$$
P\left(\nu_{a} \rightarrow \nu_{b}\right) \neq P\left(\bar{\nu}_{a} \rightarrow \bar{\nu}_{b}\right) .
$$

This is actually a well known fact - for example, the MSW effect can enhance the $\nu_{e} \leftrightarrow \nu_{\mu}$ oscillations and suppress the $\bar{\nu}_{e} \leftrightarrow \bar{\nu}_{\mu}$ ones or vice versa. Moreover, in matter the survival probabilities are not CP-invariant:

$$
P\left(\nu_{a} \rightarrow \nu_{a}\right) \neq P\left(\bar{\nu}_{a} \rightarrow \bar{\nu}_{a}\right)
$$

To disentangle fundamental CP violation from the matter induced one in the LBL experiments one would need to measure the energy dependence of the oscillated signal or the signals at two baselines, which is a difficult task. The (difficult) alternatives are:

- LBL experiments at relatively low energies and moderate baselines $(E \sim 0.1-1 \mathrm{GeV}$, $L \sim 100-1000 \mathrm{~km}$ ) 22 - in this case matter effects are negligible.

- Indirect measurements through

(A) CP-even terms $\sim \cos \delta_{\mathrm{CP}}[23$;

(B) Area of leptonic unitarity triangle [24].

CP-odd effects cannot be studied in the supernova neutrino experiments because of the experimental indistinguishability of low-energy $\nu_{\mu}$ and $\nu_{\tau}$. 


\subsection{T-odd effects in matter}

Since CPT is not conserved in matter, $\mathrm{CP}$ and $\mathrm{T}$ violations are no longer directly connected (although some relations between them still exist [21, 25]). Therefore T-odd effects in neutrino oscillation in matter deserve an independent study. Their characteristic features are:

- Matter does not necessarily induce T-odd effects (only asymmetric matter with $\tilde{N}(r) \neq$ $N(r)$ does).

- There is no $\mathrm{T}$ violation (either fundamental or matter induced) in the $2 \mathrm{f}$ case. This is a simple consequence of unitarity. For example, for the $\left(\nu_{e}, \nu_{\mu}\right)$ system one has

$$
P_{e e}+P_{e \mu}=1, \quad P_{e e}+P_{\mu e}=1,
$$

from which $P_{e \mu}=P_{\mu e}$.

- In the 3f case there is only one T-odd probability difference for $\nu$ 's (and one for $\bar{\nu}$ 's), irrespective of the matter density profile:

$$
\Delta P_{e \mu}^{T}=\Delta P_{\mu \tau}^{T}=\Delta P_{\tau e}^{T} .
$$

This is a consequence of $3 f$ unitarity [26].

The matter-induced T-odd effects are very interesting, pure $\geq 3 \mathrm{f}$ matter effects, absent in symmetric matter (in particular, in constant-density matter). They do not vanish in the regime of complete averaging of neutrino oscillations 21. They may fake the fundamental $\mathrm{T}$ violation and complicate its study, i.e. the extraction of $\delta_{\mathrm{CP}}$ from the experiment. The matter-induced T-violating effects vanish when either $U_{e 3}=0$ or $\Delta m_{21}^{2}=0$ (i.e., in the $2 \mathrm{f}$ limits) and so are doubly suppressed by both these small parameters. This implies that the perturbation theory can be used to obtain analytic expressions for the T-odd probability differences [21].

In an asymmetric matter, both fundamental and matter-induced $\mathrm{T}$ violations contribute to the T-odd probability differences $\Delta P_{a b}^{T}$. This may hinder the experimental determination of the fundamental CP- and T-violating phase $\delta_{\mathrm{CP}}$. In particular, in the accelerator LBL experiments one has to take into account that the Earth's density profile is not perfectly spherically symmetric. To extract the fundamental $\mathrm{T}$ violation, strictly speaking one would need to measure

$$
P_{\text {dir }}\left(\nu_{a} \rightarrow \nu_{b}\right)-P_{\text {rev }}\left(\nu_{b} \rightarrow \nu_{a}\right)
$$

where $P_{\text {dir }}$ and $P_{\text {rev }}$ correspond to the direct and reverse matter density profiles. (An interesting point is that even the survival probabilities $P_{\mu \mu}$ and $P_{\tau \tau}$ can be used for that 27]).

In practical terms, it would certainly be difficult to measure the quantity in (37): It would not be easy, for example, to move CERN to Gran Sasso and the Gran Sasso Laboratory to CERN. Fortunately, this is not actually necessary - matter-induced T-odd effects due to imperfect sphericity of the Earth's density distribution are very small. They cannot spoil the determination of $\delta_{\mathrm{CP}}$ if the error in $\delta_{\mathrm{CP}}$ is $>1 \%$ at $99 \%$ C.L. [21]. 


\section{3 "CPT in matter"}

As was pointed out before, a matter with unequal numbers of particles and antiparticles violates $\mathrm{CPT}$. Is there any relation between $\mathrm{CP}$ and $\mathrm{T}$ violations in matter which can play a role similar to the CPT relation in vacuum? For symmetric density profiles $(\tilde{V}(r)=V(r))$ such a relation was found in 25$]$ :

$$
P\left(\nu_{a} \rightarrow \nu_{b} ; \delta_{\mathrm{CP}}, V(r)\right)=P\left(\bar{\nu}_{b} \rightarrow \bar{\nu}_{a} ; \delta_{\mathrm{CP}},-V(r)\right)
$$

It is easy to generalize this to the case of an arbitrary density profile:

$$
P\left(\nu_{a} \rightarrow \nu_{b} ; \delta_{\mathrm{CP}}, V(r)\right)=P\left(\bar{\nu}_{b} \rightarrow \bar{\nu}_{a} ; \delta_{\mathrm{CP}},-\tilde{V}(r)\right) .
$$

Unlike CPT in vacuum, this "CPT in matter" relation does not directly relate observables (there is no anti-Earth), and so is of limited practical use. However, it can be useful for cross-checking theoretical calculations of oscillation probabilities.

\section{Why study $U_{e 3}$ ? (A hymn to $U_{e 3}$ )}

The leptonic mixing parameter $U_{e 3}$ plays a very special role in neutrino physics. It is of particular interest for a number of reasons.

First, it is the least known of leptonic mixing parameters: while we have (relatively small) allowed ranges for the other two mixing parameters, we only know an upper bound on $\left|U_{e 3}\right|$. Its smallness, which looks strange in the light of the fact that the other two mixing parameters, $\theta_{12}$ and $\theta_{23}$, are apparently large, remains essentially unexplained. (There are, however, some ideas which relate the smallness of $\left|U_{e 3}\right|$ to that of $\Delta m_{\mathrm{sol}}^{2} / \Delta m_{\mathrm{atm}}^{2}[28]$ ).

The smallness of $U_{e 3}$ is likely to be the bottleneck for studying the fundamental CP and T violation effects and matter-induced T-odd effects in neutrino oscillations. The same applies to the determination of the sign of $\Delta m_{31}^{2}$ in future LBL experiments, which would allow us to discriminate between the normal and inverted neutrino mass hierarchies. Therefore it would be vitally important to know how small $U_{e 3}$ actually is.

The parameter $U_{e 3}$ can be efficiently used to discriminate between various neutrino mass models 29]. It is one of the main parameters that drives the subdominant oscillations of atmospheric neutrinos and is important for their study. It also governs the Earth matter effects on supernova neutrino oscillations.

The parameter $U_{e 3}$ drives the parametric amplification of oscillations of core-crossing neutrinos inside the Earth, which is an interesting matter effect, different from the MSW resonance enhancement 30].

And finally, $U_{e 3}$ apparently provides us with the only opportunity to see the "canonical" MSW effect. While matter effects can be important even in the case of large vacuum mixing angles, the most spectacular phenomenon, strong enhancement of mixing by matter, can 
only occur if the vacuum mixing angle is small. From what we know now, it seems that the only small leptonic mixing parameter is $U_{e 3}$.

All this makes measuring $U_{e 3}$ one of the most important problems in neutrino physics.

\section{Conclusions}

3f effects in solar, atmospheric, reactor and supernova neutrino oscillations and in LBL accelerator neutrino experiments may be quite important. They can lead to up to 10\% corrections to the oscillation probabilities and also to specific effects, absent in the $2 \mathrm{f}$ case. The manifestations of $\geq 3$ flavours in neutrino oscillations include fundamental CP violation and $\mathrm{T}$ violation, matter-induced T-odd effects, matter effects in $\nu_{\mu} \leftrightarrow \nu_{\tau}$ oscillations, and specific CP- and T-conserving interference terms (proportional to the sines of three different mixing angles) in oscillation probabilities. The leptonic mixing parameter $U_{e 3}$ plays a very special role and its study is of great interest.

\section{References}

[1] M. C. Gonzalez-Garcia, these Proceedings, hep-ph/0410030.

[2] S. P. Mikheev, A. Y. Smirnov, Sov. J. Nucl. Phys. 42, 913 (1985) [Yad. Fiz. 42, 1441 (1985)]; L. Wolfenstein, Phys. Rev. D 17, 2369 (1978).

[3] F. J. Botella, C. S. Lim, W. J. Marciano, Phys. Rev. D 35, 896 (1987).

[4] V. D. Barger, K. Whisnant, S. Pakvasa, R. J. Phillips, Phys. Rev. D 22, 2718 (1980); H. W. Zaglauer, K. H. Schwarzer, Z. Phys. C 40, 273 (1988); T. Ohlsson, H. Snellman, J. Math. Phys. 41, 2768 (2000) (Erratum-ibid. 42, 2345 (2001)); Phys. Lett. B 474 (2000) 153; K. Kimura, A. Takamura, H. Yokomakura, Phys. Lett. B 537, 86 (2002).

[5] E. Kh. Akhmedov, R. Johansson, M. Lindner, T. Ohlsson, T. Schwetz, JHEP 0404, 078 (2004).

[6] H. Yokomakura, K. Kimura, A. Takamura, Phys. Lett. B 544, 286 (2002).

[7] E. Kh. Akhmedov, A. Dighe, P. Lipari, A.Yu. Smirnov, Nucl. Phys. B 542, 3 (1999).

[8] E. Kh. Akhmedov, Nucl. Phys. Proc. Suppl. 118, 245 (2003); Phys. Usp. 47, 114 (2004) [Usp. Fiz. Nauk 47, 121 (2004)].

[9] R. Gandhi, P. Ghoshal, S. Goswami, P. Mehta, S. U. Sankar, hep-ph/0408361. 
[10] C.-S. Lim, In Proc. BNL Neutrino Workshop on Opportunities for Neutrino Physics, Upton, N.Y., Feb. 5-7, 1987, ed. by M.J. Murtagh, Report No. BNL-52079, C87/02/05, 1987, p. 111; preprint BNL-39675 (scanned version at http://www-lib.kek.jp/cgi-bin/img_index?8710120).

[11] The CHOOZ Collaboration, M. Apollonio et al., Phys. Lett. B 420, 39 (1998); Phys. Lett. B 466, 415 (1999).

[12] M. Blennow, T. Ohlsson, H. Snellman, Phys. Rev. D 69, 073006 (2004).

[13] E. Kh. Akhmedov, M. A. Tortola, J. W. F. Valle, JHEP 0405, 057 (2004).

[14] O.L. Peres, A.Yu. Smirnov, Phys. Lett. B 456, 204 (1999).

[15] O. L. G. Peres, A. Y. Smirnov, Nucl. Phys. B 680, 479 (2004); M. C. Gonzalez-Garcia, M. Maltoni, A. Y. Smirnov, hep-ph/0408170.

[16] A. S. Dighe, A. Y. Smirnov, Phys. Rev. D 62, 033007 (2000).

[17] C. Lunardini, A.Yu. Smirnov, Phys. Rev. D 63, 073009 (2001); C. Lunardini, A.Yu. Smirnov, Nucl. Phys. B 616, 307 (2001); H. Minakata, H. Nunokawa, Phys. Lett. B 504, 301 (2001); V. Barger, D. Marfatia, B.P. Wood, Phys. Lett. B 532, 19 (2002); C. Lunardini, A. Y. Smirnov, JCAP 0306, 009 (2003).

[18] E. Kh. Akhmedov, C. Lunardini, A.Yu. Smirnov, Nucl. Phys. B 643, 339 (2002).

[19] S. Ando, K. Sato, JCAP 0310, 001 (2003).

[20] E. Kh. Akhmedov, T. Fukuyama, JCAP 0312, 007 (2003).

[21] E. Kh. Akhmedov, P. Huber, M. Lindner, T. Ohlsson, Nucl. Phys. B 608, 394 (2001).

[22] M. Koike, J. Sato, Phys. Rev. D 61, 073012 (2000) (Erratum-ibid. D 62, 079903 (2000)). H. Minakata, H. Nunokawa, Phys. Lett. B 495, 369 (2000); JHEP 0110, 001 (2001).

[23] P. Lipari, Phys. Rev. D 64, 033002 (2001).

[24] Y. Farzan, A.Yu. Smirnov, Phys. Rev. D 65, 113001 (2002); J. A. Aguilar-Saavedra, G. C. Branco, Phys. Rev. D 62, 096009 (2000); J. Sato, Nucl. Instr. Meth. A 472, 434 (2000); H. Fritzsch, Z. z. Xing, Prog. Part. Nucl. Phys. 45, 1 (2000).

[25] H. Minakata, H. Nunokawa, S. Parke, Phys. Lett. B 537, 249 (2002).

[26] P.I. Krastev, S.T. Petcov, Phys. Lett. B 205, 84 (1988).

[27] P.M. Fishbane, P. Kaus, Phys. Lett. B 506, 275 (2001). 
[28] E. Kh. Akhmedov, G.C. Branco, M.N. Rebelo, Phys. Rev. Lett. 84, 3535 (2000); S.F. King, JHEP 0209, 11 (2002); R. Kuchimanchi and R. N. Mohapatra, Phys. Lett. B 552, 198 (2003).

[29] S.M. Barr, I. Dorsner, Nucl. Phys. B 585, 79 (2000); M. Tanimoto, hep-ph/0106064 F. Feruglio, hep-ph/0410131; A. S. Joshipura, hep-ph/0411154 M. Lindner, these Proceedings.

[30] See, e.g., A. Yu. Smirnov, these Proceedings, and references therein. 